\title{
Extreme stiffness of neuronal synapses and implications for synaptic adhesion and plasticity
}

Ju Yang ${ }^{1}$, Nicola Mandriota ${ }^{1}$, Steven Glenn Harrellson ${ }^{2}$, John Anthony Jones-Molina ${ }^{1}$, Rafael

$$
\text { Yuste }^{1,3,4} \text {, Roger Lefort }{ }^{5} \text {, Ozgur Sahin }{ }^{1-3 *}
$$

${ }^{1}$ Department of Biological Sciences, Columbia University, New York, New York 10027

${ }^{2}$ Department of Physics, Columbia University, New York, New York 10027

${ }^{3}$ NeuroTechnology Center, Columbia University, New York, New York 10027

${ }^{4}$ Donostia International Physics Center, San Sebastian, Spain.

${ }^{5}$ Department of Pathology and Cell Biology, Columbia University, New York 10032

*Corresponding author: sahin@columbia.edu 


\section{Abstract}

2 Synapses play a critical role in neural circuits, and they are potential sites for learning and

3 memory. Maintenance of synaptic adhesion is critical for neural circuit function, however,

4 biophysical mechanisms that help maintain synaptic adhesion are not clear. Studies with various

5 cell types demonstrated the important role of stiffness in cellular adhesions. Although synaptic

6 stiffness could also play a role in synaptic adhesion, stiffnesses of synapses are difficult to

7 characterize due to their small size and challenges in verifying synapse identity and function. To

8 address these challenges, we have developed an experimental platform that combines atomic

9 force microscopy, fluorescence microscopy, and transmission electron microscopy. Here, using

10 this platform, we report that functional, mature, excitatory synapses had an average elastic

11 modulus of approximately $200 \mathrm{kPa}$, two orders of magnitude larger than that of the brain tissue,

12 suggesting stiffness might have a role in synapse function. Similar to various functional and

13 anatomical features of neural circuits, synaptic stiffness had a lognormal-like distribution,

14 hinting a possible regulation of stiffness by processes involved in neural circuit function. In

15 further support of this possibility, we observed that synaptic stiffness was correlated with spine

16 size, a quantity known to correlate with synaptic strength. Using established stages of the long-

17 term potentiation timeline and theoretical models of adhesion cluster dynamics, we developed a

18 biophysical model of the synapse that not only explains extreme stiffness of synapses, their

19 statistical distribution, and correlation with spine size, but also offers an explanation to how early

20 biomolecular and structural changes during functional potentiation could lead to strengthening of

21 synaptic adhesion. According to this model, synaptic stiffness serves as an indispensable

22 physical messenger, feeding information back to synaptic adhesion molecules to facilitate

23 maintenance of synaptic adhesion. 
24 Synapses are highly-specialized intercellular junctions comprised of pre- and postsynaptic

25 structures that are tightly connected by synaptic adhesion molecules ${ }^{1}$. Because synapses play a

26 critical role in learning and memory, the biochemical, morphological and electrophysiological

27 characteristics of synapses have been widely investigated. Due to their role in adhesion of pre-

28 and postsynaptic cells, mechanics of synapses can also be important for synapse function ${ }^{2}$.

29 Indeed, studies with various cell types have demonstrated the important role played by the

30 mechanical stiffness of adhering structures on the strength of cellular adhesion ${ }^{3-6}$. It could

31 similarly be the case that stiffness of synapses affects the strength of adhesion between pre-and

32 postsynaptic structures. Understanding factors that control synaptic adhesion is important due to

33 its role in maintaining connectivity and function of neural circuits. However, studying

34 mechanical characteristics of synapses are made difficult by the small sizes and complex sub-

35 structures of synapses, as well as the need for accurately identifying synapses, which require

36 biochemical and ultrastructural analysis alongside mechanical measurements.

37 We have developed an experimental platform to carry out mechanical, biochemical and

38 ultrastructural analysis of synapses by combining atomic force microscopy (AFM), fluorescence

39 microscopy and transmission electron microscopy (TEM). Although AFM has been previously

40 used to probe mechanics of synapses ${ }^{7}$, we used a recently developed cell stiffness imaging

$41 \operatorname{method}^{8}$ based on torsional harmonic AFM (TH-AFM) $)^{9,10}$ which offers high-resolution, high-

42 speed, quantitative stiffness mapping of live cells ${ }^{8}$, making the method suitable for quantifying

43 stiffness mapping of a large number of synapses. We combined this AFM method with

44 fluorescence microscopy to identify mature synapses and monitor their activity, and with TEM to

45 reliably identity synapses by observing the synaptic cleft, presynaptic vesicles, and postsynaptic

46 density, and also to correlate AFM stiffness images to synaptic ultrastructure. Although AFM is 
47 commonly used in combination with fluorescence microscopy to study biological cells,

48 combined use of AFM and TEM has been limited to imaging of filamentous biomolecules

49 deposited on substrates ${ }^{11,12}$. To achieve combined AFM and TEM imaging on the same

50 individual synapse, AFM stiffness mapping of the delicate synapses has to be performed without

51 compromising their structural integrity prior to TEM analysis. The TH-AFM allows gentle

52 probing of synapse stiffness with low peak forces during the tapping-mode AFM imaging. To

53 facilitate combined TH-AFM and TEM imaging, we developed protocols for preparing TH-

54 AFM-imaged samples for serial-sectioning, TEM imaging, and for alignment of AFM and TEM

55 data (See Methods).

56 With our experimental setup, we measured the elastic modulus of hundreds of live mature

57 excitatory synapses and mapped stiffness of postsynaptic spines and presynaptic boutons

58 identified by TEM. These experiments showed that spines are two orders of magnitude stiffer

59 than the average brain tissue. Localization of such high stiffness to a submicron structure could

60 indicate that stiffness of spines might have an important role in synaptic function. Additional

61 observations of the statistical distribution of elastic modulus values, correlation between stiffness

62 and spine size, and a mechanical model of highly crosslinked actin network provided further

63 clues to the physical origin and biochemical regulation of spine stiffness, and suggested that the

64 spine stiffness could be a quantity that adapts to the synaptic function. Based on stiffness-

65 dependent stochastic dynamics of adhesion clusters ${ }^{13,14}$, we developed a simple biophysical

66 model of the synapse that points to an indispensable role of stiffness in synapse function.

67 According to this model, functional potentiation of the synapse leads to increased stiffness,

68 which, in turn, results in a stronger synaptic adhesion. 


\section{Results}

\section{Live nanomechanical imaging of cultured neurons}

71 We characterized the nanomechanical properties of live hippocampal neurons on 14-25 days in

72 vitro (DIV) using TH-AFM (Fig. 1a). TH-AFM uses a T-shaped cantilever with an offset tip

73 (Fig. 1b) and detects both vertical and torsional deflection signals of the cantilever during

74 scanning of the sample. The vertical signal is used for the height feedback to generate the

75 topographical image and the torsional signal provides instantaneous force to calculate

76 mechanical properties during the imaging process $^{9}$ (Fig. 1c). To minimize forces acting on

77 delicate neural structures, we used low peak tapping forces (around $300 \mathrm{pN}$ ).

78 We cultured rat hippocampal neurons in glass bottom dishes and imaged live neurons in

79 Tyrode's buffer at room temperature. To capture the corresponding optical images, we placed the

80 TH-AFM apparatus above an inverted optical microscope with high magnification and high

81 numerical aperture objectives (see Methods). Hippocampal neurons were characterized as large

82 pyramidal cell bodies with long axons and branched dendritic shafts, forming complex neurite

83 networks (Fig. 1b). We optimized the culture density so that individual synapses could be

84 physically accessed by the TH-AFM tip. 
a

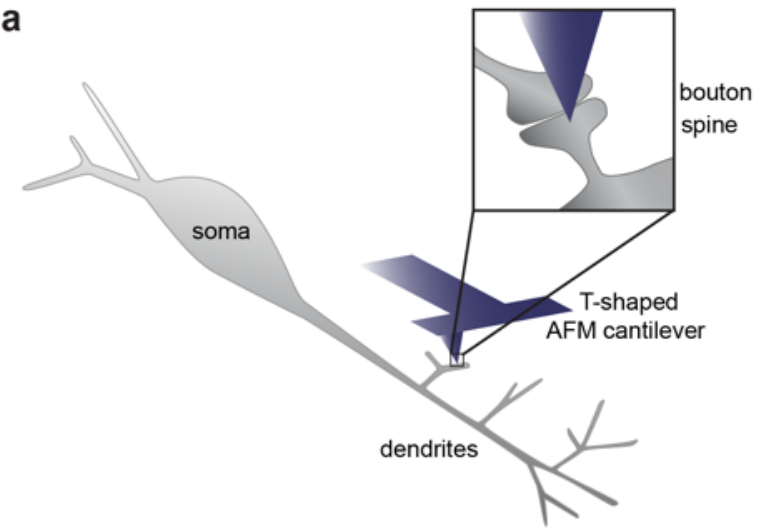

C

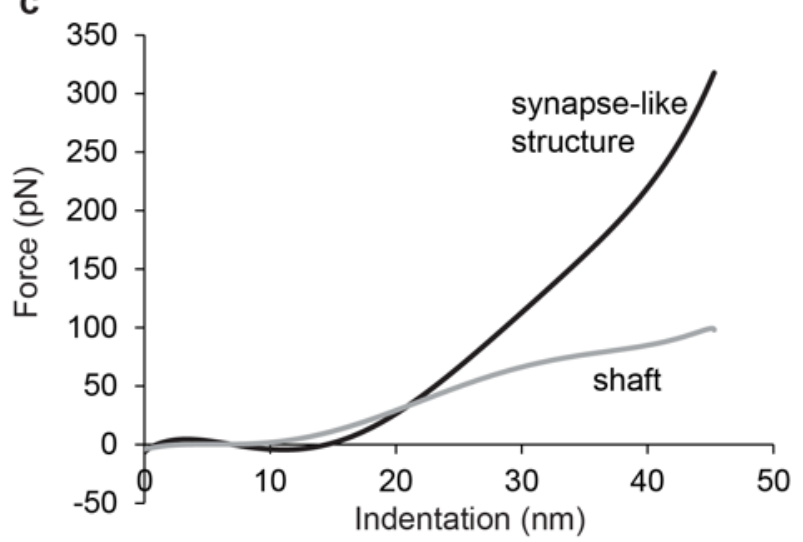

b

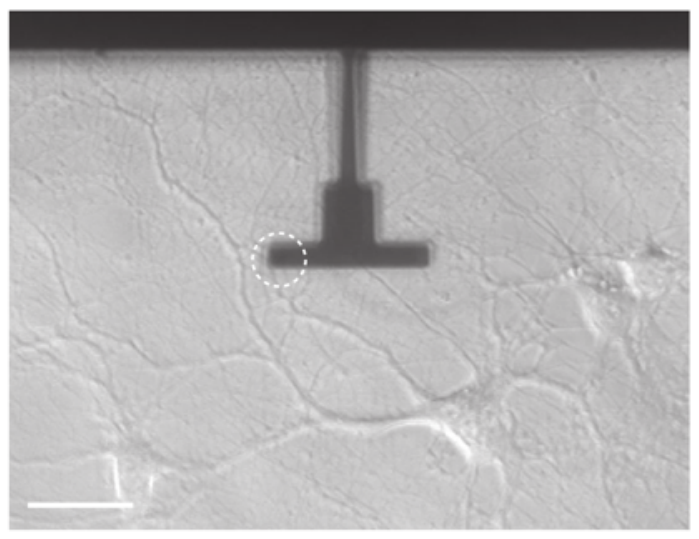

d

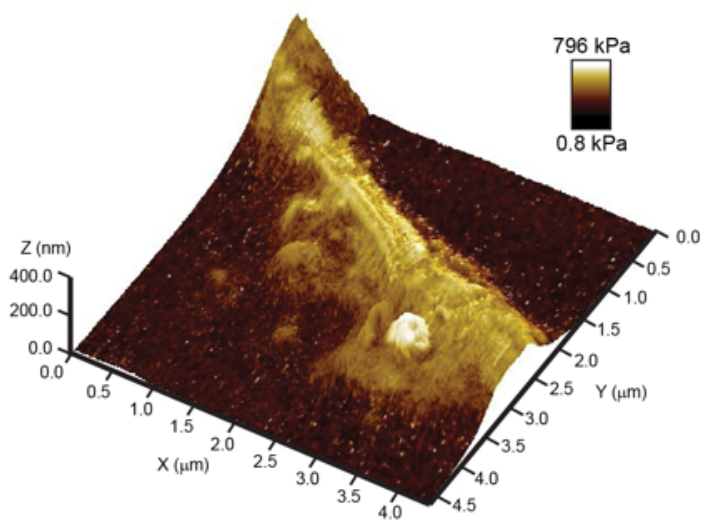

87 Figure 1: Nanomechanical imaging of live hippocampal neurons with TH-AFM.

88 a A schematic diagram illustrating the set-up of the nanomechanical imaging platform. Neurons were cultured in a glass bottom dish. TH-AFM imaging was performed by scanning a sharp tip across the sample surface while the interaction force between the tip and the sample was monitored. The zoomed-in inset illustrates the AFM tip over a synapse. b Optical image of a Tshaped cantilever over a neuron culture. The tip was facing towards the culture dish, and was placed on the left side of the cantilever (indicated by the dashed circle). Scale bar: $40 \mu \mathrm{m}$. $\mathbf{c}$ Representative force-distance curves of a synapse-like structure (black) and a dendritic shaft (grey). See Supplementary Fig. 1 for the corresponding AFM images. d Three-dimensional topographical rendering of a synapse-like structure with the color indicating elastic modulus in $\log$ scale. The elastic modulus values of the stiff structure and the shaft are $626.8 \mathrm{kPa}$ and 38.6 $\mathrm{kPa}$, respectively.

Our imaging technique generated topographical and mechanical images simultaneously during live imaging at nanometer resolution. A three-dimensional topographical rendering of a synapse-

101 like structure with the color indicating elastic modulus is shown in Fig. 1d. Within the network of processes, we observed surprisingly stiff synapse-like round structures near compliant 
103 neurites. We used the following criteria for visually-identified synapse-like structures under TH-

104 AFM: (i) distinct structures with stiffness over $20 \mathrm{kPa}$ in the mechanical image, (ii) height below

$105 \quad 1.5 \mu \mathrm{m}$ and (iii) in close proximity (within $2 \mu \mathrm{m}$ ) to a nearby neurite in the topographical image.

106 Based on these criteria, we acquired images of hundreds of synapse-like structures and nearby

107 shafts (see Methods for details of quantitative stiffness measurements), and found $77.8 \%$ of

108 synapse-like structures had a stiffness over $100 \mathrm{kPa}$. These stiff structures usually displayed a

109 round shape in topographical images and a dark contrast in optical images (Fig. 2a). Based on

110 their morphology and proximity to neurites, we hypothesized that these stiff structures could be

111 synapses.

\section{Stiff synapse-like structures are functional mature excitatory synapses}

113 To verify the identity of stiff structures revealed by TH-AFM, we performed immunostaining

114 against various synaptic markers after TH-AFM imaging and correlated immunofluorescence

115 images with AFM images. Figure 2b shows a representative stiffness image and the

116 corresponding immunostaining results of a synapse. We defined mature excitatory synapses as

117 the colocalization of the presynaptic marker Synapsin-1, and the postsynaptic marker PSD-95

118 (see Methods for colocalization detection). We found that all stiff spherical structures revealed

119 by TH-AFM were co-labeled with both Synapsin-1 and PSD-95 (Supplementary Fig. 2a, b),

120 suggesting that they were mature excitatory synapses. Interestingly, time-lapse TH-AFM

121 imaging of the same synapse showed that synapse stiffness did not vary significantly during

122 imaging (Supplementary Fig. 3). 
a
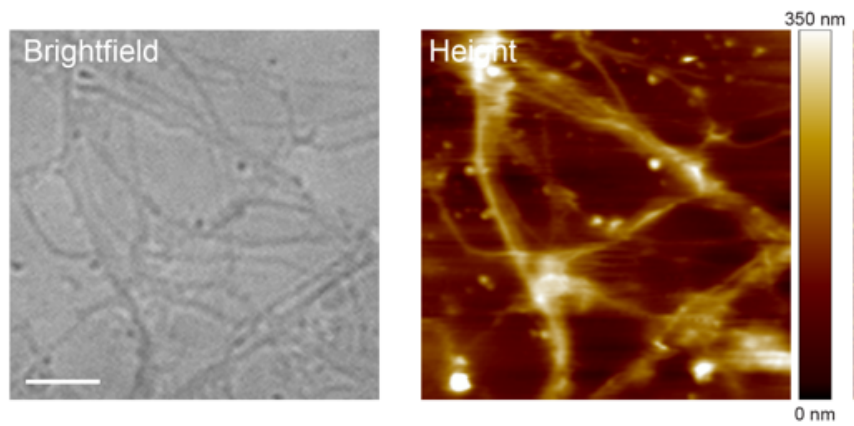

b
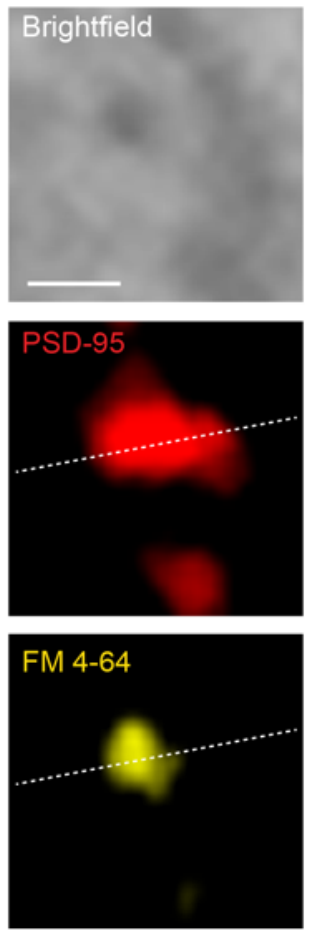
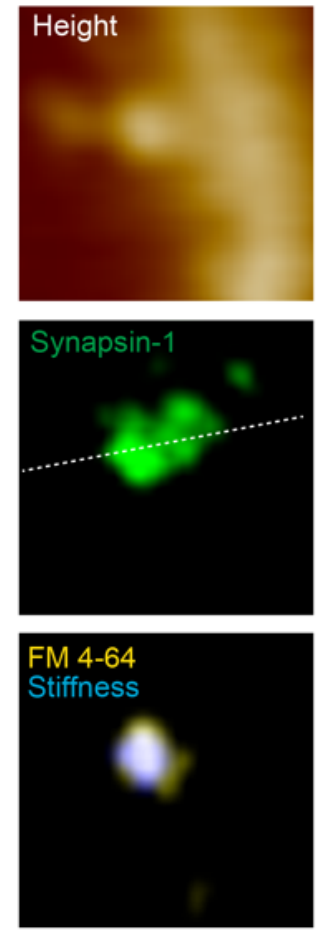
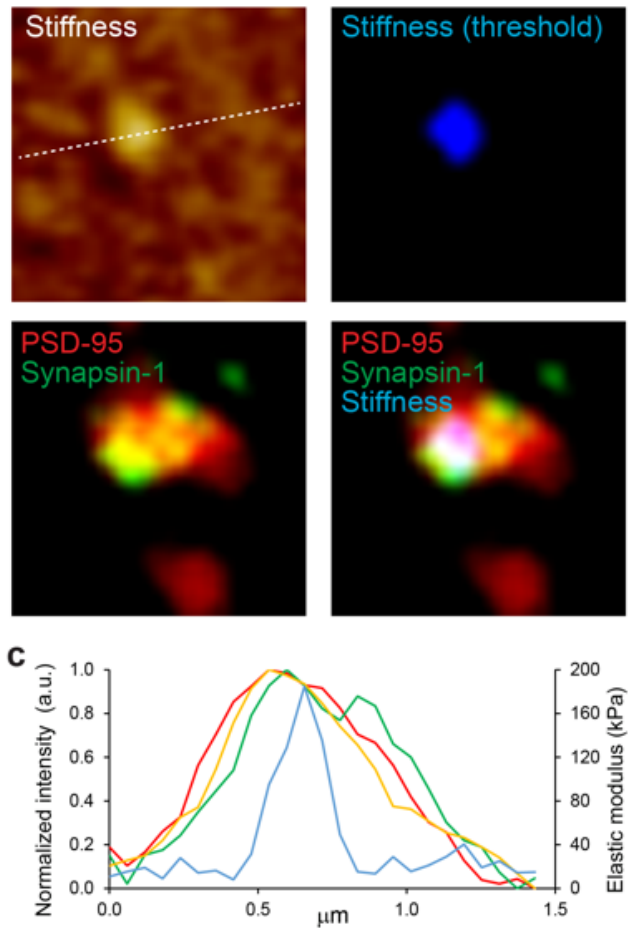

Figure 2: Correlative TH-AFM/fluorescence imaging shows stiff synapse-like structures are functional mature excitatory synapses.

126 a Aligned brightfield, AFM height, and AFM stiffness images of the same area in live neuron cultures. The color in AFM images represents height (linear scale) and elastic modulus (log scale), respectively. Scale bar: $3 \mu \mathrm{m}$. b Aligned brightfield, AFM height, AFM stiffness and fluorescence images of a representative stiff synapse from the boxed area shown in the stiffness image in a. After TH-AFM imaging, neurons were fixed and stained with postsynaptic marker PSD-95 (red) and presynaptic marker Synapsin-1 (green). Colocalization of these two markers identified mature synapses. Threshold was applied to the stiffness image colored in blue. An overlay image of stiffness, PSD-95 and Synapsin-1 shows the stiff structure was a mature synapse. In addition, neurons were stained with FM 4-64 (yellow) after TH-AFM imaging to label functional synapses. Scale bar: $500 \mathrm{~nm}$. c Fluorescence intensity and elastic modulus profiles along the dashed lines over the synapse in $\mathbf{b}$ show that high stiffness (blue) overlaps with synaptic markers, PSD-95 (red), Synapsin-1 (green), and FM 4-64 (yellow). Note that the elastic modulus has a narrower peak than fluorescence signals. 
139 To further characterize these synapses and monitor their activity, we incubated live neurons with

140 FM 4-64 dye after TH-AFM imaging (Fig. 2b, Supplementary Fig. 2c, d). FM dye binds to

141 cellular membrane and gets internalized after neurotransmitter is released, and thus only labels

142 functional presynaptic boutons ${ }^{15}$. The results show that stiff structures were colocalized with FM

143 puncta as well, indicating that these synapses were functional. Fluorescence intensity and elastic

144 modulus profiles (Fig. 2c) show that high stiffness overlaps with synaptic markers and FM dye

145 and that elastic modulus has a narrower peak than fluorescence signals. Taken together, the data

146 confirm that stiff structures identified in cultured neurons were functional and mature excitatory

147 synapses.

\section{Pre- and postsynapses have different stiffness}

149 To distinguish between pre- and postsynapses at high resolution and to investigate whether they

150 are mechanically distinct, we performed TEM imaging after TH-AFM. To locate the same

151 synapses under TEM, we cultured neurons in homemade glass bottom dishes with photoetched

152 gridded coverslips and used the numeric pattern as landmarks to align TEM images with optical

153 and AFM images ${ }^{16}$ (Supplementary Fig. 4). Serial section TEM can reveal the ultrastructure of

154 synapses at nanometer resolution ${ }^{17}$. We found that stiff structures were featured with presynaptic

155 vesicles and postsynaptic density (Fig. 3 and Supplementary Fig. 5). In particular, spines, rather

156 than boutons, overlapped with high stiffness pixels. We conclude that the high stiffness observed

157 under TH-AFM originated from postsynaptic spines and that postsynaptic spines are

158 mechanically different from presynaptic boutons. 
a
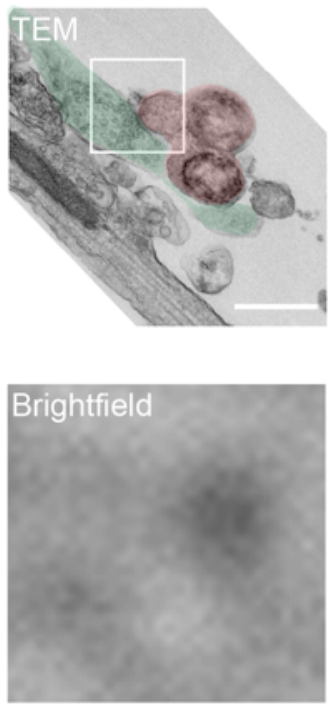

b

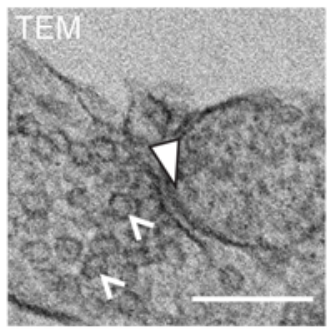

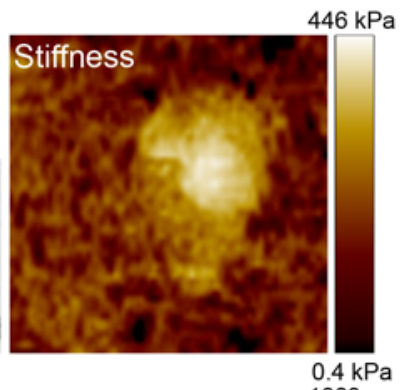

$0.4 \mathrm{kPa}$
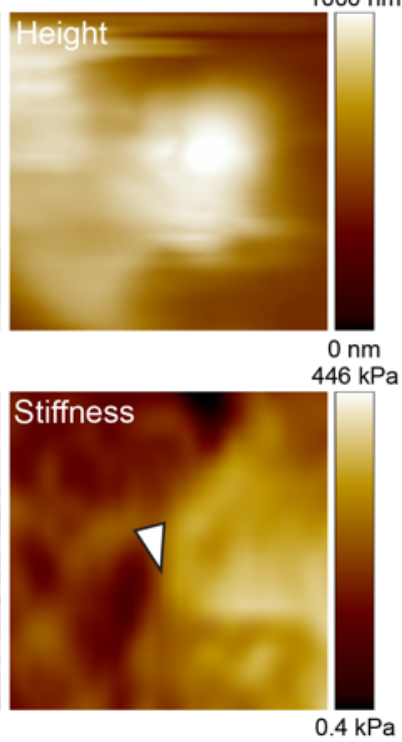

160 Figure 3: Correlative TH-AFM/TEM reveals ultrastructure of stiff synapses.

161 a Aligned TEM, AFM stiffness, brightfield, and AFM height images of the same synapse. We

162 used the numeric pattern on photoetched coverslips as landmarks to align TEM images with

163 optical and AFM images. In the TEM image, the bouton is shaded in green and the spine is

164 shaded in red. High stiffness in the AFM stiffness image overlapped mostly with the spine head

$165(209 \mathrm{kPa})$, while the bouton and dendritic shaft showed lower stiffness. The stiff spine displayed

166 distinct contrast in the optical brightfield image and topographical features in the AFM height

167 image. Scale bar: $500 \mathrm{~nm}$. b Zoomed-in TEM and AFM stiffness images of the boxed area

168 shown in the TEM image in a illustrates the synaptic cleft. White arrowheads point to the

169 postsynaptic density. White carets point to presynaptic vesicles. Scale bar: $200 \mathrm{~nm}$.

\section{Spines are substantially stiffer than shafts}

171 Intriguingly, the stiffness of spines fell within a wide range. The stiffness of shafts, on the

172 contrary, was constantly low (Fig. 4a). Spines were on average 10 times stiffer than nearby

173 shafts. The minimum, maximum, median, and mean elastic modulus values of spines are 23.2 
$174 \mathrm{kPa}, 671.9 \mathrm{kPa}, 166.9 \mathrm{kPa}$ and $201.3 \mathrm{kPa}$, whereas the minimum, maximum, median, and mean

175 elastic modulus values of shafts are $7.1 \mathrm{kPa}, 67.4 \mathrm{kPa}, 20.7 \mathrm{kPa}$ and $23.6 \mathrm{kPa}$ (see Methods). The

176 distributions of spine and shaft stiffness were strongly skewed with heavy tails and exhibited the

177 characteristics of a lognormal distribution. We measured the apparent spine size from AFM

178 stiffness images by thresholding the stiffness signal to identify region of interest for area

179 measurement (see Methods). We found that the distribution of apparent spine size was also

180 skewed with a heavy tail (Fig. 4b). Spine stiffness measurements were positively correlated with

181 apparent spine size (Fig. 4c) (see Supplementary Notes for additional discussion about the

182 sensitivity of the correlation analysis to the contact mechanics models), suggesting that larger

183 spines may have underlying changes in actin architecture ${ }^{18}$. In addition, we found that immature

184 protrusions, likely filopodia, were not stiff (Supplementary Fig. 6), suggesting that stiffness may

185 increase as spines mature, and possibly change with synaptic function and activity. The

186 surprisingly high stiffness of spines may represent a unique parameter related to synaptic activity

187 and function that is complementary to the well-established biochemical and electrophysiological

188 parameters.

189 Our criteria for synapse-like structures under TH-AFM might exclude spiny synapses with a

190 stiffness below $20 \mathrm{kPa}$ or without distinct height features in topographical images (for example,

191 spines buried underneath neurites), and shaft synapses that are formed directly on the shaft

192 without protrusive spine structures. Nevertheless, given that the measured spine stiffness had a

193 lognormal-like distribution with a peak around $164 \mathrm{kPa}$, very few spines would be excluded by

194 our criteria. 
a

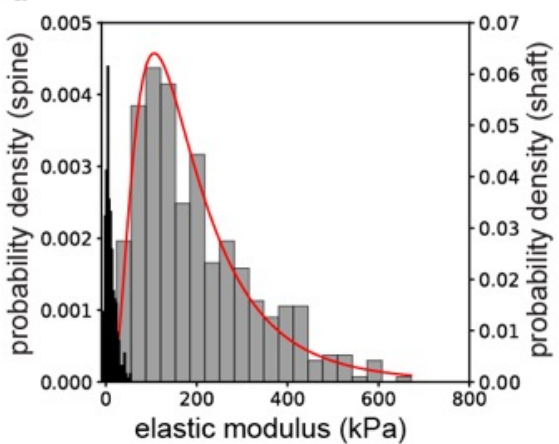

b

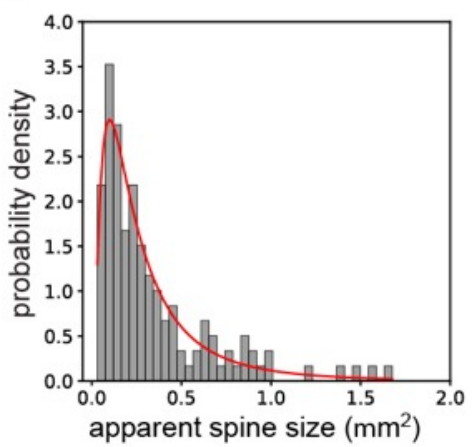

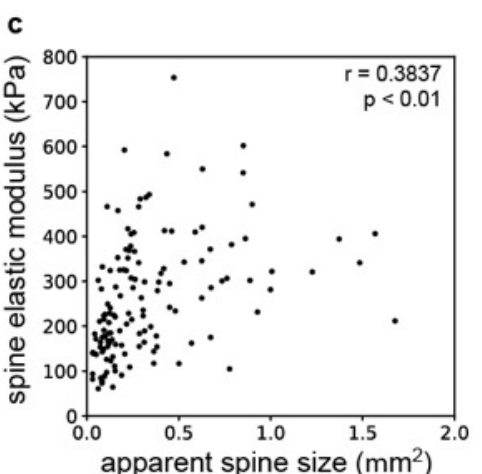

196

197

198

199

200

201

202

203

204

205

206

207

208

209

210

211

212

213

214

215

216

217

Figure 4: Spine stiffness is higher than shaft stiffness, and is correlated with spine size.

a Histogram of elastic modulus of spines (grey) and nearby shafts (black) with bins $=20 . n=409$ spines / 30 neuron cultures. We fitted the logarithm data with a normal distribution (spine stiffness fitted curve shown here in red). The mean elastic modulus values of spines and shafts from the fitting are $164.3 \mathrm{kPa}$ and $21.4 \mathrm{kPa}$, respectively. We tested the goodness of fit using Kolmogorov-Smirnov tests $\left(p_{\text {spine }}=0.3192, p_{\text {shaft }}=0.2446\right)$. $\mathbf{b}$ Histogram of the apparent spine size measured from AFM stiffness images with bins $=37 . \mathrm{n}=134$ spines $/ 20$ neuron cultures. We fitted the logarithm of data with a normal distribution (red fitted curve with a mean spine size of $0.23 \mu \mathrm{m}^{2}$.) and tested the goodness of fit using a Kolmogorov-Smirnov test $(\mathrm{p}=0.9486)$.

c Correlation of spine elastic modulus with apparent spine size from $\mathbf{b}$. r: Pearson correlation coefficient. $p$ : significance of correlation with a two-tailed $t$ test. $p=4.719 \mathrm{E}-06$.

\section{Mechanical synaptic plasticity}

The average value of elastic modulus of spines $(201.3 \mathrm{kPa})$ is approximately two orders of magnitude larger than the average elastic modulus values reported for the gray and white matter brain tissue ${ }^{19}$. The substantially high stiffness of spines suggests a potential novel physiological role. Stiffness could be necessary to maintain spine morphology in the presence of synaptic adhesion. The unique spine morphology with an enlarged head and a thin neck enables compartmentalization of chemical and electrical signals, which is critical for synaptic function and plasticity ${ }^{20}$. Treating the synapse as two soft bodies in adhesive contact suggests that spine stiffness has to be on the order of $100 \mathrm{kPa}$ to maintain its shape (see modeling and calculations in Supplementary Notes and Supplementary Table $1-4$ ), which is consistent with the measured values of spine stiffness in Fig. 4a. However, the stiffness requirement for maintaining spine 
218 morphology only specifies an approximate minimum value for stiffness, therefore it would not

219 lead to a particular distribution of stiffness values, nor would it predict a positive correlation with

220 spine size, which are clearly observed in our measurements in Fig. 4.

221 A potentially critical role for stiffness in synapse function could be due to the connection

222 between stiffness and cell adhesion, which could explain both our quantitative measurements and

223 the observed distributions and correlations. Previous studies of the stochastic dynamics of

224 adhesion molecules ${ }^{13}$ clustered at the site of adhesion has shown that the overall lifetime of a

225 cluster depends on the stiffness of adhering surfaces ${ }^{14}$. In this view, due to the stochastic nature

226 of molecular interactions, adhesion bonds rupture and rebind continuously. When bonds are

227 ruptured, the surfaces can deform due to a small but non-zero force that pulls the surfaces apart.

228 As illustrated in Fig. 5a, rupture of bound molecules will increase the distance between those

229 molecules, reducing the probability of their future rebinding, and thereby decreasing the lifetime

230 of the adhesion cluster. When the surfaces get stiffer, the probability of rebinding increases,

231 leading to stabilization of the adhesion cluster. Gao et al. ${ }^{14}$ showed that a typical adhesion cluster

232 is substantially stabilized as the sample stiffness increases beyond 50 to $100 \mathrm{kPa}$ (Fig. 5b). The

233 stiffness-dependent lifetime of adhesion clusters could also be applicable to synaptic adhesion,

234 because pre- and postsynapses are connected together by transmembrane synaptic adhesion

235 molecules ${ }^{1}$. Indeed, one of these molecules, N-cadherin, has been shown to be strengthened on

236 stiffer substrates in C2 mouse myogenic cells ${ }^{6}$. Importantly, our measured spine elastic modulus

237 values in Fig. 4a correspond to the regime where the lifetime of adhesion would be greatly

238 enhanced, as shown in Fig. 5b. 
a

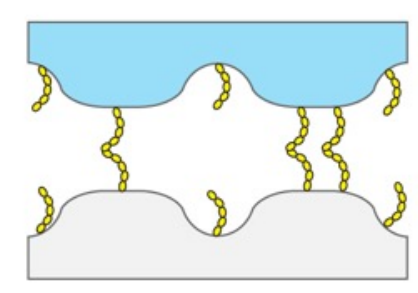

b

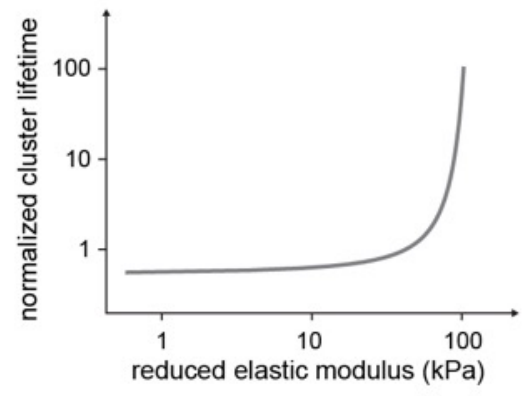

C

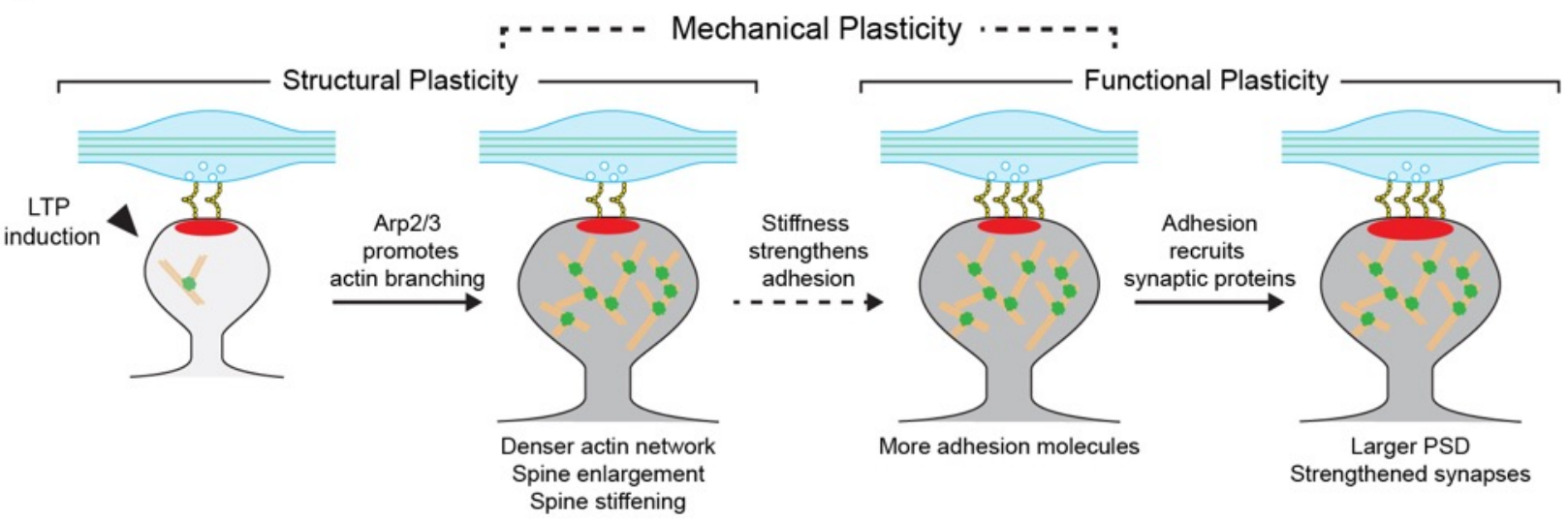

Figure 5: Mechanical synaptic plasticity.

a Two elastic bodies (blue and grey) are connected by a cluster of adhesion molecules (depicted in yellow). Following the rupture of bonds between individual molecules, elastic recoil reduces the probability of rebinding due to the separation of binding molecules, causing stochastic dynamics of the adhesion cluster. $\mathbf{b}$ The lifetime of a cluster increases drastically as the sample stiffens. Diagrams in $(\mathbf{a}, \mathbf{b})$ are adapted from Qian and $\mathrm{Gao}^{14}$. $\mathbf{c}$ The mechanical synaptic plasticity model based on the stiffness-dependent stochastic dynamics of adhesion clusters. The schematic illustrates a simplified sequence of synaptic changes during LTP. The postsynaptic spine (grey) and the presynaptic axonal bouton (blue) are physically connected by synaptic adhesion molecules such as N-cadherin (yellow). Spine with postsynaptic density (PSD; red) has enriched actin networks (orange) cross-linked by Arp2/3 (green), while axon contains rigid microtubules (green lines). During LTP, spine exhibits both structural plasticity and functional plasticity with spine enlargement, actin reorganization, PSD enlargement, and increase of synaptic transmission. As shown by the dashed arrow, increased stiffness during structural plasticity (indicated by the darkening grey color of the spine) could enhance the adhesion at the synapse by increasing the surface density of bound adhesion molecules at the synapse. Adhesion molecules then help recruit synaptic proteins including PSD-95, AMPAR, and NMDAR, resulting in PSD enlargement and functional potentiation. Upon subsequent stimulations, a strengthened spine would have more $\mathrm{Ca}^{2+}$ influx, causing further spine stiffening and thus creating a positive feedback to maintain spine stiffness and synaptic potentiation. 
259 Given the connection between stiffness and adhesion strength, we propose a biophysical model,

260 the mechanical plasticity model, to explain how stiffness can be enhanced during long-term

261 potentiation (LTP), and how the enhanced stiffness can facilitate maintenance of synaptic

262 adhesion (Figure 5c). This role of spine stiffness fits well into the previously established LTP

263 timeline: During LTP, NMDAR-dependent $\mathrm{Ca}^{2+}$ signaling cascade leads to structural plasticity

264 involving spine enlargement and actin remodeling ${ }^{21,22}$, followed by an increase in adhesion

265 molecules such as N-cadherin ${ }^{23}$, followed by enlargement of the postsynaptic density (PSD) ${ }^{18,24}$,

266 and later an increase in synaptic receptors and enhanced synaptic strength. The structural

267 changes that occur early in the LTP timeline, particularly the actin remodeling can lead to a

268 substantial increase in the stiffness of actin network, which, in turn, would strengthen the

269 synaptic adhesion.

270 High resolution imaging methods show that spines contain densely crosslinked actin

271 networks ${ }^{25,26}$ (see also Supplementary Fig. 7), which could explain the origin of high stiffness of

272 spines. To test this possibility quantitatively, we used a simple mechanical model of a three-

273 dimensional network of fibers that relates the elastic modulus of the network to the geometry of

274 the network with the following relationship ${ }^{27}$ :

$$
E_{N} \approx E_{F}\left(\frac{a}{L}\right)^{4}
$$

276 Here $E_{N}$ is the elastic modulus of fiber network, $E_{F}$ is the elastic modulus of individual fibers, $a$

277 is fiber radius, and $L$ is the distance between nodes of the network where fibers are connected. In

278 the case of a crosslinked actin network, $a$ would correspond to the nominal radius of actin

279 filaments, and $L$ would correspond to the average distance between crosslinks. Using and $a \approx$ 
$3.5 \mathrm{~nm}$ and $E_{F} \approx 1 \mathrm{GPa}$ for the nominal radius and elastic modulus ${ }^{28}$ of F-actin filaments, Eq. (1) predicts that the average distance between crosslinks of the actin network of the spine to be in

282 the range of 22 to $53 \mathrm{~nm}$ to match the experimentally observed elastic modulus values (i.e. 20 to

$283600 \mathrm{KPa})$. These number agree with electron microscopy images of actin networks in spines ${ }^{26}$

284 that show distances below about 50 nanometers, judged by comparing network dimensions to the

285 diameter of individual actin filaments, $\sim 7 \mathrm{~nm}$. Consequently, this analysis suggests that spine

286 stiffness originates from the cross-linking of actin architecture, which can be due to Arp2/3

287 complexes $^{29}$.

288 Importantly, because actin remodeling during LTP follows the NMDAR-dependent $\mathrm{Ca}^{2+}$

289 signaling cascade, crosslink-dependent spine stiffness can be expected to correlate with spine

290 morphological changes and synaptic strength, which would explain the observed correlation with

291 spine size (Fig. 4a) and the lognormal-like distribution of spine stiffness (Fig. 4c), as synaptic

292 strength is known to follow a lognormal-like distribution ${ }^{30}$.

293 Taken together, the extreme stiffness of synapses, attributable to the extensive crosslinking of the

294 postsynaptic F-actin network, along with the biophysical model summarized in Fig. 5, points to

295 an indispensable role stiffness could be playing in synapses: a physical messenger that conveys

296 information about LTP-driven biomolecular and structural changes in the postsynaptic spine to

297 the synaptic adhesion molecules, resulting in stronger synaptic adhesion. This potential coupling

298 of information from the physical domain (stiffness) back to biochemical interactions (adhesion

299 molecules) could link structural plasticity to functional plasticity ${ }^{31}$, and help maintain synaptic

300 connectivity. 


\section{Methods}

302

303

304

305

306

307

308

309

\section{Hippocampal neuron culture preparation}

Animal work was approved by the Columbia University Institutional Animal Care and Use Committee. Hippocampal neuron cultures were prepared following a modified version of the previously described Brewer method ${ }^{32}$. Fetuses at embryonic day 18 (E18) from timed pregnant Sprague-Dawley rats (Taconic Farms; Hudson, NY) were sacrificed and the hippocampi removed and collected in room temperature Hank's balanced salt solution (HBSS-; Thermo Fisher Scientific 14025076), supplemented with 0.6\% (w/v) glucose (HBSS+). The hippocampi were then incubated in $0.05 \%$ trypsin (Thermo Fisher Scientific 25300054) for 15 minutes at $37^{\circ} \mathrm{C}$ and washed with $\mathrm{HBSS}+$ three times for 10 minutes each. Finally, the neurons were dissociated in Neurobasal medium (Thermo Fisher Scientific 21103-049) supplemented with B27 supplements (Thermo Fisher Scientific 17504-044) and 0.5 mM L-Glutamine (Thermo Fisher Scientific 25030). Neurons were plated at a density of 100,000 cell $/ \mathrm{mL}$ in glass bottom dishes coated with $1 \mathrm{mg} / \mathrm{mL}$ poly-L-lysine (Sigma-Aldrich P2636) and $10 \mu \mathrm{g} / \mathrm{mL}$ mouse protein laminin (Thermo Fisher Scientific 23017-015). 50 mm glass bottom dishes (WillCo GWSt-5040) were used in most experiments unless otherwise specified. The resulting neuronal cultures consisted of a population enriched in large pyramidal neurons. Cultures were maintained in 5\% $\mathrm{CO}_{2}$ humidified incubator at $37^{\circ} \mathrm{C}$ and used after $14-25$ days in vitro (DIV) to image mature synapses, and DIV 5-7 to image immature protrusions. Before imaging, culture medium was replaced with Tyrode's buffer ( $125 \mathrm{mM} \mathrm{NaCl}, 2 \mathrm{mM} \mathrm{KCl}, 3 \mathrm{mM} \mathrm{CaCl}, 1 \mathrm{mM} \mathrm{MgCl} 2-6 \mathrm{H}_{2} \mathrm{O}, 10$ mM HEPES, $30 \mathrm{mM}$ D-glucose, adjusted to $300 \mathrm{mOsm}$ with sucrose, $\mathrm{pH}$ adjusted to 7.4 with $\mathrm{NaOH})^{33}$ at room temperature. Neuronal activities were verified with calcium indicator Oregon Green (Thermo Fisher Scientific O6807) showing neuron firing. 


\section{Torsional harmonic cantilevers}

325 T-shaped cantilevers were custom made ${ }^{8}$ by Bruker-Nano, Inc with the following specifications:

326 the cantilever bodies were made of silicon nitride and the tip was made of silicon. The length,

327 width, and thickness of the cantilevers were nominally $85 \mu \mathrm{m}, 9 \mu \mathrm{m}$, and $650 \mathrm{~nm}$. The width at

328 the free end was $60 \mu \mathrm{m}$, and the tip offset $25 \mu \mathrm{m}$, tip height $5 \mu \mathrm{m}$ or $6.5 \mu \mathrm{m}$. Cantilevers were

329 coated with silicon nitride via plasma-enhanced chemical vapor deposition to a radius of $75 \mathrm{~nm}$ or

$330100 \mathrm{~nm}$. Flexural and torsional deflection sensitivities of cantilevers were determined from ramp

331 plots, assuming flexural and torsional motions to be described by springs in series. The spring

332 constants of flexural (approximately $0.2 \mathrm{~N} / \mathrm{m}$ ) and torsional (approximately $1.0 \mathrm{~N} / \mathrm{m}$ ) deflections

333 were determined from the respective thermal noise spectra.

\section{Atomic force microscopy imaging}

335 Glass bottom dishes were mounted on the stage of an inverted fluorescence microscope Axio

336 Observer Z1 (Zeiss) and neurons were perfused with Tyrode's buffer during imaging. TH-AFM

337 experiments were performed with BioScope Catalyst (Bruker) and imaging was carried out in

338 fluid tapping mode. T-shaped cantilevers were analyzed in real time to create topographical and

339 mechanical maps as previously described ${ }^{9}$. The set point amplitude was approximately $45 \mathrm{~nm}$

340 and the tip-sample interaction force was approximately $300 \mathrm{pN}$. Elastic modulus was calculated

341 by fitting the force-distance curves with a Derjaguin-Muller-Toporov (DMT) model $^{34}$ with a

342 hemispherical indenter (see Supplementary Methods for additional discussion about elastic

343 modulus calculation). All AFM images were recorded with 512 pixel $\times 256$ pixel, 256 pixel $\times$

344256 pixel, or 256 pixel $\times 128$ pixel over areas of $5 \mu \mathrm{m} \times 5 \mu \mathrm{m}$ to $25 \mu \mathrm{m} \times 25 \mu \mathrm{m}$ with a scan

345 rate 0.5 to $1.5 \mathrm{~Hz}$. 


\section{Atomic force microscopy data analysis}

347 AFM data were processed and analyzed with NanoScope Analysis 1.70 (Bruker), SPIP 5.0

348 (Image Metrology), Gwyddion, and ImageJ. For quantitative measurement, in order to reduce

349 noise, a $3 \times 3$ median filter was applied to stiffness images. Due to edge effect, AFM

350 measurement is more reliable on top of a structure close to the center, and less so close to the

351 edge. Thus, we selected areas of interest $(\mathrm{AOI})$ of at least 10 pixel $\times 10$ pixel close to the center

352 on a spine structure identified by the topographical image, and used the maximum value in the

353 AOI to represent the elastic modulus of a spine. We noticed that the stiffness of a dendritic shaft

354 and an immature protrusion did not vary substantially along its length. We thus drew a section

355 line of at least 10 pixels along the length of a shaft structure or an immature protrusion close to

356 its centerline, and used the average value along the section to represent the elastic modulus of a

357 shaft and a filopodium, respectively. For apparent spine size measurement, we applied a median

358 filter to the AFM stiffness image. Substrate signal was measured by selecting a substrate region

359 in the image and plotting a Gaussian distribution histogram showing mean $\mu$ and standard

360 deviation $\sigma$. The threshold was then set as $\mu+3 \sigma$ to identify AOI for area measurement. For

361 intensity profile in Fig. 2c, raw elastic modulus data were used. For visualization purpose only, a

362 low pass filter was applied to height images; spike removal with vertical interpolation and local

363 mean equalization were applied to stiffness images.

\section{Epifluorescence microscopy}

365 Optical images were taken before and after TH-AFM imaging using an inverted microscope

366 (Axio Observer Z1; Zeiss) at different magnifications (10X, 20X, 100X EC Plan-Neofluar,

367 Zeiss). We used phase contrast at $10 \mathrm{X}$ and $20 \mathrm{X}$ and brightfield at $100 \mathrm{X}$ in optical imaging. After

368 live imaging, the location of neurons of interest was marked by labeling the relative position of 
the perfusor (Bruker) to the dish, and the relative position of perfusor to the objective to ensure

370 the same regions could be captured after immunocytochemistry. Fluorescence images were taken

371 using the same epifluorescence microscope (Axio Observer Z1; Zeiss) with proper filter sets

372 (Zeiss and Chrome) at 100X magnification (1.3 NA). All images were captured with a standard

373 CCD camera (Hamamatsu) at 1344 pixel $\times 1024$ pixel resolution.

\section{Functional labeling of presynaptic boutons with FM 4-64}

375 At the end of the TH-AFM experiment, neurons were incubated in high KCl Tyrode's buffer (77

$376 \mathrm{mM} \mathrm{NaCl}, \mathrm{KCl} 50 \mathrm{mM}, 3 \mathrm{mM} \mathrm{CaCl} 2,1 \mathrm{mM} \mathrm{MgCl} 2-6 \mathrm{H}_{2} \mathrm{O}, 10 \mathrm{mM}$ HEPES, $30 \mathrm{mM}$ D-glucose,

377 adjusted to 300 mOsm with sucrose, $\mathrm{pH}$ adjusted to 7.4 with $\mathrm{NaOH}$ ) with $10 \mu \mathrm{M}$ FM 4-64 for 45

378 seconds $^{35}$, and then washed with calcium-free Tyrode's buffer $(125 \mathrm{mM} \mathrm{NaCl}, 2 \mathrm{Mm} \mathrm{KCl}, 1 \mathrm{mM}$

$379 \mathrm{MgCl}_{2}-6 \mathrm{H}_{2} \mathrm{O}, 10 \mathrm{Mm}$ HEPES, $30 \mathrm{mM}$ D-glucose, adjusted to $300 \mathrm{mOsm}$ with sucrose, $\mathrm{pH}$

380 adjusted to 7.4 with $\mathrm{NaOH}$ ) for 15 minutes to remove non-specific membrane bound FM 4-64.

381 After FM 4-64 imaging, cells were washed with normal Tyrode's buffer for 30 minutes to

382 remove trapped FM dyes, before being proceeded to immunocytochemistry.

\section{Immunocytochemistry}

384 The primary antibodies used were PSD-95 (1:1,000, mouse; Abcam ab99009) and Synapsin-1

385 (1:1,000, rabbit; Cell Signaling 5297). The secondary antibodies used were Alexa Fluor® 488

386 Goat Anti-Mouse (1:5000, Thermo Fisher Scientific A-11029), Alexa Fluor ${ }^{\circledR} 488$ Goat Anti-

387 Rabbit (1:5000, Thermo Fisher Scientific A-11034), Alexa Fluor ${ }^{\circledR} 546$ Goat Anti-

388 Mouse(1:5000, Thermo Fisher Scientific A-11030), Alexa Fluor ${ }^{\circledR} 546$ Goat Anti-Rabbit

389 (1:5000, Thermo Fisher Scientific A-11035), Cy5® Goat Anti-Mouse (1:5000, Thermo Fisher

390 Scientific A10524), Cy5® Goat Anti-Mouse (1:5000, Thermo Fisher Scientific A10523). To 
391 label F-actin, Alexa Fluor ${ }^{\circledR} 546$ Phalloidin (1:500, Thermo Fisher Scientific A22283) was added

392 to the secondary antibody solution. After TH-AFM imaging, neurons were fixed with 4\% (w/v)

393 paraformaldehyde (Thermo Fisher Scientific 28908), permeabilized with $0.3 \%$ (v/v) Triton X-

394100 (Sigma-Aldrich 93443) in phosphate-buffered saline (PBS), and incubated with primary

395 antibodies diluted in SuperBlock Blocking Buffer (Thermo Fisher Scientific 37515) overnight at

$3964^{\circ} \mathrm{C}$. Neurons were then incubated with secondary antibodies at room temperature for 30 minutes

397 to 2 hours.

\section{Sample preparation for TEM}

399 To acquire the ultrastructure of synapses, neurons were cultured in homemade glass bottom

400 dishes (Corning ${ }^{\circledR}$ 60mm TC-Treated Culture Dish 430166) on gridded coverslips (Electron

401 Microscopy Sciences 72264-18 and 72265-50). After live optical and TH-AFM imaging,

402 neurons were fixed in the dish with $2.5 \%(\mathrm{w} / \mathrm{v})$ glutaraldehyde in $0.15 \mathrm{M}$ sodium cacodylate

403 buffer $(\mathrm{pH} 7.4)$ at room temperature for 1 hour and then at $4^{\circ} \mathrm{C}$ overnight. Neurons were then

404 rinsed 3 times in $0.1 \mathrm{M}$ cacodylate buffer at $4^{\circ} \mathrm{C}$ and post-fixed with $1 \% \mathrm{OsO}_{4}$ in $0.1 \mathrm{M}$

405 cacodylate buffer at $4^{\circ} \mathrm{C}$ for 1 hour. After block staining with $1 \%$ uranyl acetate at $4^{\circ} \mathrm{C}$ for 1

406 hour, neurons were rinsed 3 times with $\mathrm{ddH}_{2} \mathrm{O}$ at $4^{\circ} \mathrm{C}$ and dehydrated in a gradient of ethanol:

$40730 \%, 50 \%, 70 \%$ at $4^{\circ} \mathrm{C}$ for 5 minutes each, $85 \%, 95 \%$ at room temperature for 5 minutes each,

408 and $100 \%$ four times for 5 minutes each. Neurons were infiltrated in 100\% ethanol/Araldite 502

409 (Electron Microscopy Sciences 13900) at room temperature: 1:1 twice for 10 minutes each, 1:2

410 for 10 minutes, and 100\% Araldite three times for 10 minutes each, then $100 \%$ Araldite

411 overnight. The sample was flat embedded and polymerized at $60^{\circ} \mathrm{C}$ for 48 hours. 


\section{Serial section TEM}

413 The sample block was detached from the coverslip by immersing the whole dish in liquid

414 nitrogen, and then trimmed under stereoscope. The grid pattern imprinted in the resin served as

415 landmarks. $70 \mathrm{~nm}$ serial ultrathin sections were cut using Leica UC6 ultramicrotome (Leica

416 Microsystems Inc., Buffalo Grove, IL), collected on formvar coated slot grids, and stained with

417 uranyl acetate and lead citrate. The top few sections containing the marker grid pattern were

418 recognized under transmission electron microscope (Philips CM-12, FEI, Eindhoven, The

419 Netherland) at lower magnification (170X), and were used to locate the regions of interest based

420 on the comparison of neurites morphology from optical images. To identify regions of interest in

421 deeper sections, the relative location of regions of interest on TEM sections was marked on the

422 captured images (Gatan 4k×2.7k digital camera, Gatan Inc., Pleasanton, CA) and used as

423 reference. Serial sections of neurites and synapses were then imaged at 170X - 66000X

424 magnification.

\section{Image processing}

426 Optical images were processed and quantified with ImageJ and Caltracer 2 (available through:

427 http://blogs.cuit.columbia.edu/rmy5/methods/). Background signal was measured by selecting

428 dark regions in an image and plotting a Gaussian distribution histogram showing mean $\mu$ and

429 standard deviation $\sigma$. The intensity of the whole image was then subtracted by $\mu+3 \sigma$ to remove

430 noise. Subtraction processed images were then analyzed using Caltracer to identify

431 colocalization. Puncta contours of each marker were detected using an automated algorithm

432 based on fluorescence intensity, puncta size, and shape, and were adjusted by visual inspection.

433 Contours of different markers (PSD-95 and Synapsin-1) were overlaid and a threshold of 5\%

434 overlap was used for all potential colocalization detection. Colocalized contours were counted 
435 and overlaid with stiffness images. TEM images were processed with the FIJI plugin Enhance

436 Local Contrast (CLAHE) (available through:

437 http://imagej.net/Enhance_Local_Contrast_(CLAHE)) to enhance local contrast for visualization,

438 with histogram bins 50. Optical images, AFM images, and TEM images of different

439 magnifications of the same area were aligned in Adobe Photoshop and visually inspected. In

440 particular, due to the non-linear lens distortions induced by the electromagnetic lenses of TEM,

441 serial section TEM images were usually distorted. In order to align TEM images with optical

442 images and AFM images, we skewed the TEM images with shear transformation in Adobe

443 Photoshop. For visualization purpose only, brightness and contrast was adjusted, median filter

444 was applied, and pixel number was increased to smoothen the pixelated images in Adobe

445 Photoshop. For quantitative analysis including intensity profiles, raw data were used unless

446 otherwise specified.

\section{Statistical analysis}

448 Error bars in all figures represented standard error of mean. Two-tailed tests were used in

449 statistical analysis. Sample size (n), p value, and Pearson correlation coefficient (r) were given in

450 figure legends when applicable. Each neuron culture represented an independent experiment. A

451 significance level of 0.01 was used in hypothesis tests and $\mathrm{p}<0.01$ was considered significant.

452 To fit the transformed data to a normal distribution, the maximum likelihood estimation method

453 was used. Goodness of fit tests of the transformed data in a normal distribution were performed

454 using two-tailed Kolmogorov-Smirnov tests with the null hypothesis that the transformed data is

455 normally distributed. We tested goodness of fit for different transformations including logarithm,

456 square root, and cubic root, as well as for non-transformed data (see Supplementary Table 5 for $\mathrm{p}$

457 values). Given that for spine stiffness $(\mathrm{kPa})$, shaft stiffness $(\mathrm{kPa})$, spine size $\left(\mu \mathrm{m}^{2}\right)$, logarithm 
458 transformation has the highest $\mathrm{p}$ value (the probability of the null hypothesis being true), the data

459 is likely to follow a lognormal-like distribution. All data were analyzed using Python.

\section{Data availability}

461 The data supporting the findings of this study are available within the paper and the

462 Supplementary Information. The remaining data are available from the corresponding author on 463 request.

\section{Acknowledgements}

465 We thank NYU Microscopy Core, Kristen Dancel-Manning, Chris Petzold, and Alice Liang, for 466 their assistance in TEM, and Reka Recinos and Hardik Patel for neuronal cultures. This work 467 was supported by the NIH Director's New Innovator Award Program (1DP2-EB018657), the 468 NIMH (R01MH101218, R01MH100561), NINDS (R01NS110422; R34NS116740), and the U.

469 S. Army Research Laboratory and the U. S. Army Research Office under contract number 470 W911NF-12-1-0594 (MURI).

\section{Author Contributions}

472 J.Y. carried out AFM imaging, fluorescence microscopy imaging, and TEM imaging, contributed

473 to the conceptualization, cantilever preparation, improvement of imaging protocols, data

474 analysis, and development of mechanical models. N.M. demonstrated proof of principle for the

475 AFM imaging in neurons and contributed to the development of imaging protocols. S.G.H.

476 contributed to the development of mechanical models and data analysis. J.J.M. contributed to

477 AFM and fluorescence microscopy imaging, and cantilever preparation. R.Y. contributed to the 
478 conceptualization, experimental design, and discussions. R.L. prepared neuron cultures,

479 contributed to the conceptualization, experimental design, and discussions. O.S. contributed to

480 the conceptualization, supervision, cantilever design, development of mechanical models, and

481 data analysis. J.Y. and O.S. wrote the manuscript with input from all coauthors.

\section{Competing interests}

483 Authors declare no competing financial interests.

\section{References}

1 Dalva, M. B., McClelland, A. C. \& Kayser, M. S. Cell adhesion molecules: signalling functions at the synapse. Nat Rev Neurosci 8, 206-220, doi:10.1038/nrn2075 (2007).

2 Tyler, W. J. The mechanobiology of brain function. Nature Reviews Neuroscience 13, 867878, doi:10.1038/nrn3383 (2012).

3. Wang, N, Butler, J.P., \& Ingber, D.E. Mechanotransduction across the cell surface and through the cytoskeleton Science, 260, 1124-1127 (1993) .

4. Choquet, D., Felsenfeld, D.P., \& Sheetz, M.P. Extracellular matrix rigidity causes strengthening of integrin-cytoskeleton linkages Cell, 88, 39-48 (1997).

5 Guo, W., Frey, M.T., Burnham, N.A., \& Wang, Y. Substrate Rigidity Regulates the Formation and Maintenance of Tissues. Biophysical J. 90, 2213-2220 (2006).

6 Ladoux, B. et al. Strength Dependence of Cadherin-Mediated Adhesions. Biophys J 98, 534542, (2010). doi:10.1016/j.bpj.2009.10.044.

7 Smith, B. A., Roy, H., De Koninck, P., Grutter, P. \& De Koninck, Y. Dendritic spine viscoelasticity and soft-glassy nature: Balancing dynamic remodeling with structural stability. Biophys J 92, 1419-1430, doi:10.1529/biophysj.106.092361 (2007).

8 Mandriota, N., Friedsam, C., Jones-Molina, J. A, Tatem, K.V., Ingber, D. \& Sahin, O. Cellular nanoscale stiffness patterns governed by intracellular forces. Nat. Mater. 18, 1071-1077 doi:10.1038/s41563-019-0391-7 (2019)

9 Sahin, O., Magonov, S., Su, C., Quate, C. F. \& Solgaard, O. An atomic force microscope tip designed to measure time-varying nanomechanical forces. Nat Nanotechnol 2, 507-514, doi:10.1038/nnano.2007.226 (2007).

10 Dong, M. D., Husale, S. \& Sahin, O. Determination of protein structural flexibility by microsecond force spectroscopy. Nat Nanotechnol 4, 514-517, doi:10.1038/Nnano.2009.156 (2009).

11 Lin, A.C. \& Goh, M.C. A novel sample holder allowing atomic force microscopy on transmission electron microscopy specimen grids: repetitive, direct correlation between 
AFM and TEM images. Journal of Microscopy, 205, 205-208 (2002). doi:10.1046/j.0022-

2720.2001.00978.x

12 Yamada, Y., Konno, H., \& Shimabukuro. K. Demonstration of correlative atomic force and transmission electron microscopy using actin cytoskeleton. Biophys. Physicobiol. 14, 111117 (2017). doi:10.2142/biophysico.14.0_111

13 Erdmann, T. \& Schwarz, U.S. Stochastic dynamics of adhesion clusters under shared constant force with rebinding. J. Chem. Phys. 121, 8997, doi: 10.1063/1.1805496 (2004)

14 Qian, J. \& Gao, H. Soft matrices suppress cooperative behaviors among receptor-ligand bonds in cell adhesion. PLoS One 5, e12342, doi:10.1371/journal.pone.0012342 (2010).

15 Ryan, T. A. et al. The Kinetics of Synaptic Vesicle Recycling Measured at Single Presynaptic Boutons. Neuron 11, 713-724, doi:10.1016/0896-6273(93)90081-2 (1993).

16 Reddick, L. E. \& Alto, N. M. Correlative Light and Electron Microscopy (CLEM) as a Tool to Visualize Microinjected Molecules and their Eukaryotic Sub-cellular Targets. Jove-J Vis Exp, doi:10.3791/3650 (2012).

17 Darcy, K. J., Staras, K., Collinson, L. M. \& Goda, Y. An ultrastructural readout of fluorescence recovery after photobleaching using correlative light and electron microscopy. Nat Protoc 1, 988-994, doi:10.1038/nprot.2006.146 (2006).

18 Bosch, M. et al. Structural and molecular remodeling of dendritic spine substructures during long-term potentiation. Neuron 82, 444-459, doi:10.1016/j.neuron.2014.03.021 (2014).

19 Budday S. et al. Mechanical properties of gray and white matter brain tissue by indentation. J. Mech. Behav. Biomed. Mater. 46, 318-330 doi:10.1016/j.jmbbm.2015.02.024 (2015)

20 Yuste, R. \& Bonhoeffer, T. Morphological changes in dendritic spines associated with longterm synaptic plasticity. Annu Rev Neurosci 24, 1071-1089, doi:10.1146/annurev.neuro.24.1.1071 (2001).

21 Matsuzaki, M., Honkura, N., Ellis-Davies, G. C. \& Kasai, H. Structural basis of long-term potentiation in single dendritic spines. Nature 429, 761-766, doi:10.1038/nature02617 (2004).

22 Okamoto, K., Nagai, T., Miyawaki, A. \& Hayashi, Y. Rapid and persistent modulation of actin dynamics regulates postsynaptic reorganization underlying bidirectional plasticity. Nat Neurosci 7, 1104-1112, doi:10.1038/nn1311 (2004).

23 Bozdagi, O., Shan, W., Tanaka, H., Benson, D. L. \& Huntley, G. W. Increasing numbers of synaptic puncta during late-phase LTP: N-cadherin is synthesized, recruited to synaptic sites, and required for potentiation. Neuron 28, 245-259 (2000).

24 Meyer, D., Bonhoeffer, T. \& Scheuss, V. Balance and stability of synaptic structures during synaptic plasticity. Neuron 82, 430-443, doi:10.1016/j.neuron.2014.02.031 (2014).

25 Nagerl, U. V., Willig, K. I., Hein, B., Hell, S. W. \& Bonhoeffer, T. Live-cell imaging of dendritic spines by STED microscopy. Proc Natl Acad Sci U S A 105, 18982-18987, doi:10.1073/pnas.0810028105 (2008).

26 Korobova, F. \& Svitkina, T. Molecular architecture of synaptic actin cytoskeleton in hippocampal neurons reveals a mechanism of dendritic spine morphogenesis. Mol. Biol. 
27 Gibson, LC and Ashby, MF, Cellular Solids: Structure and Properties. Pergamon Press, Oxford 1988.

28 Gardel, M. L., Kasza, K. E., Brangwynne, C. P., Liu, J., \& Weitz, D. A. Mechanical response of cytoskeletal networks. Methods in cell biology, 89, 487-519. (2008). https://doi.org/10.1016/S0091-679X(08)00619-5

29 Spence, E. F., Kanak, D. J., Carlson, B. R. \& Soderling, S. H. The Arp2/3 Complex Is Essential for Distinct Stages of Spine Synapse Maturation, Including Synapse Unsilencing. J Neurosci 36, 9696-9709, doi:10.1523/JNEUROSCI.0876-16.2016 (2016).

30 Buzsaki, G. \& Mizuseki, K. The log-dynamic brain: how skewed distributions affect network operations. Nature Reviews Neuroscience 15, 264-278, doi:10.1038/nrn3687 (2014).

31 Straub, C. \& Sabatini, B. L. How to Grow a Synapse. Neuron 82, 256-257, doi:10.1016/j.neuron.2014.03.033 (2014).

32 Brewer, G. J., Torricelli, J. R., Evege, E. K. \& Price, P. J. Optimized Survival of HippocampalNeurons in B27-Supplemented Neurobasal(Tm), a New Serum-Free Medium Combination. J Neurosci Res 35, 567-576, doi:DOI 10.1002/jnr.490350513 (1993).

33 Kralj, J. M., Douglass, A. D., Hochbaum, D. R., Maclaurin, D. \& Cohen, A. E. Optical recording of action potentials in mammalian neurons using a microbial rhodopsin. Nat Methods 9, 90-U130, doi:10.1038/Nmeth.1782 (2012).

34 Derjaguin, B. V., Muller, V. M. \& Toporov, Y. P. Effect of Contact Deformations on the Adhesion of Particles. Prog Surf Sci 45, 131-143, doi:10.1016/0079-6816(94)90044-2 (1994).

35 Gaffield, M. A. \& Betz, W. J. Imaging synaptic vesicle exocytosis and endocytosis with FM dyes. Nat Protoc 1, 2916-2921, doi:10.1038/nprot.2006.476 (2006) 